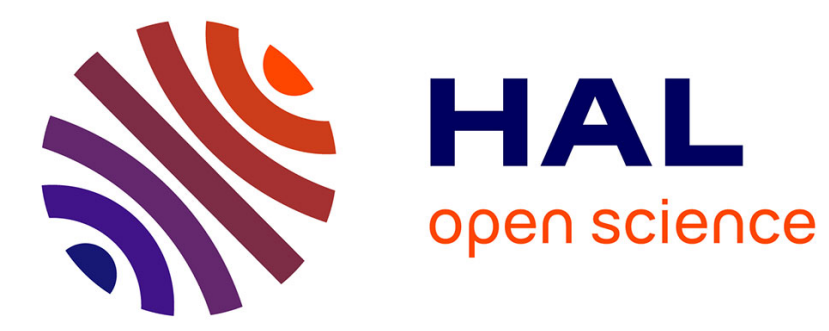

\title{
Quel(s) statut(s) pour les sociaux-démocrates est-allemands (1989-1990)?
}

Etienne Dubslaff

\section{To cite this version:}

Etienne Dubslaff. Quel(s) statut(s) pour les sociaux-démocrates est-allemands (1989-1990)? Allemagne d'aujourd'hui: revue francaise d'information sur l'Allemagne, 2017, 220, pp.14-24. 10.3917/all.220.0014 . hal-03185360

\section{HAL Id: hal-03185360 https://hal.science/hal-03185360}

Submitted on 21 May 2021

HAL is a multi-disciplinary open access archive for the deposit and dissemination of scientific research documents, whether they are published or not. The documents may come from teaching and research institutions in France or abroad, or from public or private research centers.
L'archive ouverte pluridisciplinaire HAL, est destinée au dépôt et à la diffusion de documents scientifiques de niveau recherche, publiés ou non, émanant des établissements d'enseignement et de recherche français ou étrangers, des laboratoires publics ou privés.

\section{(이) $\$$}

Distributed under a Creative Commons Attribution - NonCommercial - NoDerivatives $\mid 4.0$ 


\section{Quel(s) statut(s) pour les sociauxdémocrates est-allemands (1989-1990) ?}

"Les questions de statuts sont toujours des questions de pouvoir ${ }^{1}$. Cette assertion du père des statuts successifs du Parti social-démocrate en République démocratique allemande (Sozialdemokratische Partei in der Deutschen Demokratischen Republik, SDP puis SPD), Konrad Elmer, paraît bien banale. Et pourtant, ces questions revêtent un intérêt tout particulier durant la courte période d'existence de cette formation politique, du 7 octobre 1989 au 27 septembre 1990, qui voit l'élaboration de trois textes différents. Parallèlement, le parti passe d'un groupuscule d'une quarantaine de membres à une organisation revendiquant entre 30000 et 41500 membres $^{2}$, d'un parti d'opposition fondamentale au régime quarantenaire de la RDA à un parti de gouvernement après la tenue des élections libres du 18 mars 1990, enfin, d'une entité jalouse de son indépendance vis-à-vis des autres formations politiques à une partie intégrante du SPD de RFA. Ces évolutions internes au parti accompagnent, accélèrent et reflètent tout autant les profondes mutations que connaît la RDA durant la même période : jusqu'au 9 novembre 1989, l'État est-allemand vit à l'ombre du Mur mais connaît un bouillonnement révolutionnaire certain que le SDP ne fait qu'amplifier. II provoque la chute d'Erich Honecker le 18 octobre puis de son successeur Egon Krenz le 3 décembre, la fin progressive du régime autoritaire ${ }^{3}$ par l'abrogation de l'article

* Maître de conférences à l'Université Paul-Valéry Montpellier 3, rattaché au Centre de recherche et d'études germaniques (CREG)

1. ELMER, Konrad, "Vor- und Wirkungsgeschichte des Organisationsstatuts der SDP », in : DOWE, Dieter / ECKERT, Rainer (éd.), Von der Bürgerbewegung zur Partei. Die Gründung der Sozialdemokratie in der DDR. Diskussionsforum im Berliner Reichstag am 7. Oktober 1992, coll. Gesprächskreis Geschichte, $n^{\circ} 3$, Forschungsinstitut der Friedrich-Ebert-Stiftung, Bonn, 1993, p. 29-39, p. 29.

2. Cf. STURM, Daniel Friedrich, Uneinig in die Einheit. Die Sozialdemokratie und die Vereinigung Deutschlands 1989/90, Willy-Brandt-Studien, Dietz, Bonn, 2006, p. 269 et HILSBERG, Stephan, « Liebe Parteifreunde ! », Berlin, 10 juillet 1990 , in : [AdsD] 2/SDPA000168 (Archiv der sozialen Demokratie).

3. Sur les débats historiographiques autour de la nature du régime, cf. notamment KOTT, Sandrine, "La RDA dans la recherche historique allemande. De la "Seconde dictature" à l'histoire politique au quotidien ", in : Matériaux pour I'histoire de notre temps, t. 68, $\mathrm{n}^{\circ} 1,2002$, p. 24-29, LÜDTKE, Alf, « La République démocratique allemande comme histoire. Réflexions historiographiques », in : Annales Histoire Sciences Sociales, janvierfévrier 1998, vol. 53, n 1 / 1998, EHESS, Paris, p. 3-39 et COURTOIS, Stéphane / POMIAN, Krzysztof, « 1989 : pourquoi la désintégration du bloc soviétique ? Un échange ", in : Revue Le Débat, NovembreDécembre 2009, Gallimard, Paris, 2009, p. 3-15.

premier de la constitution le $1^{\text {er }}$ décembre ${ }^{1}$ et l'instauration de la Table ronde centrale qui doit assurer la transition vers les élections libres une semaine plus tard. Celles-ci balaient définitivement les tenants du Nationale Front et propulsent le dorénavant SPD-Est au gouvernement. Dès décembre 1990, les sociaux-démocrates est-allemands se confrontent

\footnotetext{
1. "La République démocratique allemande est un État socialiste de nation allemande. Elle est l'organisation politique des travailleurs à la ville comme à la campagne qui réalisent le socialisme conjointement sous la direction de [la] classe ouvrière et de son Parti marxiste-léniniste. ", cf. DÜRIG, Günter / RUDOLF, Walter (éd.), Texte zur deutschen Verfassungsgeschichte, Rechtshistorische Texte, Beck'sche Verlagsbuchhandlung, Munich, 1996, p. 294-319, p. 294.
} 
à la question allemande ${ }^{2}$ et se rapprochent de ce qui devient progressivement leur homologue de Bonn, le SPD-Ouest. Au-delà du 18 mars 1990, interprété quasi unanimement comme un plébiscite en faveur de l'unification nationale, ils sont en outre amenés à préparer parallèlement la fusion des deux États et des deux partis sociaux-démocrates allemands ${ }^{3}$.

Les textes statutaires d'octobre 1989, de février et de septembre 1990, analysés comme autant de miroirs des évolutions du parti et de la RDA - si ce n'est de l'Allemagne -, nous permettront de comprendre ce que signifie être social-démocrate en RDA durant la dernière année d'existence de l'État est-allemand, à tout le moins en ce qui concerne les membres de la première heure, les seuls à avoir un impact certain sur l'élaboration des trois textes ${ }^{4}$.

\section{Le parti contre le Parti}

Lorsque les deux initiateurs de la création du SDP, les pasteurs Markus Meckel et Martin Gutzeit, exposent leurs visées à un parterre non moins protestant et intellectuel le 26 août 1989 , leur projet est bel et bien de fonder un parti. Le public leur réserve un accueil mitigé. Cette forme d'organisation est décriée par l' « opposition » qui entend en combattre les abus en privilégiant le contre-modèle, celui des mouvements dits citoyens qui se développent depuis le milieu des années 1980 en RDA et foisonnent à l'été $1989^{5}$. D'ailleurs, un an plus tôt, Meckel et Gutzeit envisageaient encore de créer l'association « Participation citoyenne "6. À l'été 1989, Meckel et Gutzeit ont acquis, comme la plupart de ceux qui les rejoignent jusqu'en octobre, une expérience du fonctionnement des mouvements citoyens et se montrent pour le moins réservés quant à leur efficacité politique. Pour eux, ils servent avant tout de soupape de sécurité à des personnes qui peuvent y critiquer le régime relativement librement parce qu'ils sont placés sous l'aile protectrice de l'Église protestante ${ }^{7}$. Faute d'affiliation pérenne, les réunions ne débouchent pas sur une feuille de route que des élus auraient à charge de mettre en application. Au mieux, les mouvements se cantonnent à

2. Par un communiqué de presse, le Directoire reconnaît que la question de l'unification se pose mais revendique qu'elle se fasse entre partenaires égaux. La partie programmatique des "Statut 1 " reconnaissait quant à elle l'unité de la nation et l'existence de deux États allemands, cf. VORSTAND DER SDP, "SDP zur Deutschlandfrage ", Berlin, 3 décembre 1989, in : [RHG] SDP02. La cote renvoie aux archives de la Société Robert Havemann.

3. Cf. TESSMER, Carsten, Innerdeutsche Parteienbeziehungen vor und nach dem Umbruch in der DDR, Institut für Gesellschaft und Wissenschaft (IGW) an der Universität Erlangen-Nürnberg, Deutsche Gesellschaft für zeitgeschichtliche Fragen e.V., Erlangen, 1991, p. 53 et p. 57 et KORTE, Karl-Rudolf, Die Chance genutzt ? Die Politik zur Einheit Deutschlands, Campus Verlag, Francfort sur le Main / New York, 1994, p. 124 et p. 209.

4 . I nversement, les échelons subalternes contribuent activement à étoffer le programme, du moins jusqu'en janvier 1990. Il est vrai qu'en cela, ils répondent aux appels du Directoire national qui publie une sélection de textes en amont de la Conférence des délégués (" Delegiertenversammlung »), cf. BOGISCH, Frank, PAWLICZAK, Lothar (éd.), Querschnitt. Denkmodelle zur künftigen Wirtschafts-, Umwelt-, und Sozialpolitik der Sozialdemokratischen Partei in der DDR, Berlin, [10 janvier] 1990, [RHG] CL 12.

5. Le 21 août 1989, R. Eppelmann, F. Schorlemmer, E. Richter et W. Schnur fondent le Renouveau démocratique (Demokratischer Aufbruch, DA), le 9 septembre, le Nouveau Forum (Neues Forum, NF) est créé par K. Havemann, B. Bohley, M. Böttger et J. Reich puis, trois jours plus tard, la Démocratie maintenant (Demokratie Jetzt !, DJ) voit le jour à l'initiative d'Ulrike Poppe. La plupart de ces personnages clé de la " révolution pacifique ", tout comme Meckel et Gutzeit du reste, milite depuis 1985 dans I'Initiative pour la paix et les Droits de l'Homme (Initiative Frieden und Menschenrechte, IFM), premier réseau important d'opposants au régime, cf. MUSIOLEK, Berndt / WUTTKE, Carola (dir.), Parteien und politische Bewegungen im letzten Jahr der DDR, BasisDruck Verlagsgesellschaft, Berlin, 1991, p. 2755.

6. MECKEL / GUTZEIT, « Das Vereinsprojekt "Bürgerbeteiligung" (Juni 1988) », in : MECKEL, Markus / GUTZEIT, Martin, Opposition in der DDR. Zehn Jahre kirchliche Friedensarbeit - kommentierte Quellentexte, Bund-Verlag, Cologne, 1994, p. 355 sq.

7. Sur le rôle de l'Église protestante comme pépinière des mouvements d'opposition, cf. KOWALCZUK, Ilko-Sascha, Endspiel. Die Revolution von 1989 in der DDR, BPB, Bonn, 2009, p. 197-204. 
revendiquer des aménagements, là où la création d'un parti défie ouvertement le pouvoir en place. Justement, les deux pasteurs entendent renverser un régime qu'ils vivent - tout comme les mouvements du reste-comme dictatorial ${ }^{8}$. Pour eux, il s'agit de construire une entité politique qui vise à conquérir le pouvoir pour appliquer un programme défini en amont ${ }^{9}$. Le fait même de se doter de structures fixes, d'agréger des militants engagés dans la durée qui délèguent le pouvoir à des mandataires élus et responsables devant leur base, revient à proposer un contre-modèle méthodique au sein de l'opposition. Se revendiquer de la social-démocratie ne fait que renforcer la dynamique révolutionnaire puisque cela vient délibérément remettre en cause les assises mythiques du Parti socialiste unifié (Sozialistische Einheitspartei Deutschlands, SED), issu de la fusion du Parti communiste d'Allemagne (Kommunistische Partei Deutschlands, KPD) et du Parti social-démocrate d'Allemagne (Sozialdemokratische Partei Deutschlands, SPD) dans la zone d'occupation soviétique en $1946^{10}$. Le fait de créer un tel parti, dont l'illégalité assumée rompt de surcroît avec le légalisme dont font preuve les mouvements citoyens ${ }^{11}$, nie l'unité de la classe ouvrière au sein du SED et sape, dans le contexte mythique de la RDA, le fondement de son pouvoir. La création effective, le 7 octobre 1989 dans un presbytère du village brandebourgeois de Schwante, tandis que le régime fête en grande pompe le quarantième anniversaire de son existence à BerlinEst, constitue une provocation supplémentaire à l'égard du Parti ${ }^{12}$. Aussi convient-il d'interpréter la fondation du parti social-démocrate en RDA comme doublement révolutionnaire : révolutionnaire à l'égard du SED, mais aussi révolutionnaire du point de vue de la méthode contestataire vis-à-vis des mouvements. Dans les deux cas, l'existence même de statuts contraignants fonde cette puissance potentielle du SDP. Venons-en à l'étude des texte-cadre à proprement parler pour dégager les emprunts aux deux formes d'organisation mais aussi les traits proprement originaux du SDP/ SPD-Est qui en fondent l'identité.

Les premiers statuts ("Statut 1 ») appelés à jeter les bases du fonctionnement du parti - sans être contraignants au niveau national - sont adoptés sans débats le 7 octobre $1989^{13}$. Plus précisément, Meckel argue de ce que des discussions prolongées feraient planer le risque d'une intervention de la Stasi avant la fondation du parti ${ }^{14}$. L'étude des deux textes liminaires (« Entwurf 1 » et « Entwurf 2 ») permet de reconstituer les positions en présence

8. Cf. MECKEL, « Programmatischer Vortrag zur Gründung der Sozialdemokratischen Partei in der DDR (SDP) am 7. Oktober 1989 in Schwante ", in : MECKEL / GUTZEIT, Opposition in der DDR..., op. cit., p. 379-396, p. 381, et entretien avec Meckel, Berlin, le 22 mars 2011. Les entretiens mentionnés font référence à ceux menés par l'auteur dans le cadre de la thèse de doctorat inédite "Oser plus de social-démocratie ". La création et l'établissement du Parti socialdémocrate en RDA.

9. Cette définition correspond à celle avancée par ARMINGEON, Klaus : " Parteien, Verbände und soziale Bewegungen », in : MÜNKLER, Herfried (dir.), Politikwissenschaft. Ein Grundkurs, coll. Rowohlts Enzyklopädie, Rowohlt Taschenbuch Verlag, Reinbek bei Hamburg, 2003, p. 447-489, p. 448 sq.

10. Cf. MALYCHA, Andreas / WINTERS, Peter Jochen, Geschichte der SED. Von der Gründung bis zur Linkspartei, BPB, Bonn, 2009, p. 35.

11. Cf. ANSONG, Leonore / GEHRKE, Bernd / KLEIN, Thomas / KNEIPP, Thomas (dir.) : " Das Land ist still - noch ! ". Herrschaftswandel und politische Gegnerschaft in der DDR (1971-1989), Zeithistorische Studien, t. 40, Böhlau Verlag, Vienne / Cologne / Weimar, 2009, p. 26.

12. Le ministre de la Stasi, Erich Mielke, ne s'y trompe pas, cf. HEIMANN, Siegfried, « SDP-Gründung am 7. Oktober 1989. Vortrag am 7. Oktober 1999 in Berlin-Pankow », in : KREISVERBAND DER SPD-PANKOW (éd.), Wir 89er. 20 Jahre Sozialdemokratie in Pankow, Weißensee und Prenzlauer Berg, SPD-Pankow, Berlin, 2009, p. 7-14, p. 13.

13. "Statut der SDP - Sozialdemokratische Partei in der DDR - SDP », in : [RHG] SDP 01.

14. Cf. WEIDEN, " Das Profil der Sozialdemokratischen Partei in der DDR (SDP/SPD) », in : SCHUH, Petra / WEIDEN, Bianca M. von der, Die deutsche Sozialdemokratie 1989 / 90. SDP und SPD im Einigungsproze $\beta$, Schriftreihe der Forschungsgruppe Deutschland, $n^{\circ}$ 9, Forschungsgruppe Deutschland, Munich, 1997, p. 13-180, p. 51.

15. Cf. ELMER, Konrad / KAISER, Klaus-Dieter / RÜHLE, Reiner, « SDP - Sozialdemokratische Partei in der DDR. Vorläufiges Statut. Entwurf 1 » ["Entwurf 1 »], daté du 20 septembre et ELMER / KAISER, « SDP - Sozialdemokratische Partei in 
sinon parmi tous les membres fondateurs, du moins au sein du groupe de préparation de la fondation ${ }^{16}$. Notons à cet égard que Konrad Elmer s'est emparé de la problématique réglementaire car il favorisait une structure conforme à la république des conseils telle que la prônaient Rosa Luxembourg et Hannah Arendt ${ }^{17}$.

L'originalité de ces premiers textes réside en l'alliance entre la démocratie représentative avec des mandats forts mais exclusifs - le cumul de mandats au sein du parti et de l'État est expressément interdit - et la volonté de créer de petites structures d'une quinzaine de membres à tous les niveaux qui délèguent à chaque fois deux des leurs à l'échelon supérieur. Les pères des premiers statuts entendent garantir à chaque membre la possibilité de participer activement aux débats et donc à la vie du parti. Celui-ci doit devenir l'instrument qui permet à la population de sortir de la passivité dans laquelle elle s'est laissé enfermer par le régime ${ }^{18}$. La volonté de favoriser le débat, déjà essentielle pour les mouvements citoyens, doit déboucher, pour les sociaux-démocrates, sur l'émergence de militants citoyens ${ }^{19}$. Cet ethos socialdémocrate reste une spécificité est-allemande que les représentants de Berlin tentent d'imposer au parti ouest-allemand dans le cadre de la fusion.

Autrement moins typique du fonctionnement des mouvements citoyens, le pouvoir du mandataire se renforce au gré des premières ébauches. En réaction à la longévité en poste, caractéristique des élus du SED, les premières versions disposaient encore qu'un même membre ne saurait exercer les mêmes fonctions au-delà de deux ans. Les "Statut 1 ", quant à eux, suppriment cette limitation tout en instaurant un gardefou : une reconduction audelà de huit ans nécessiterait le vote favorable des deux tiers des militants électeurs. II en va de même en matière de révocation d'un élu, rendue plus difficile en ce que la majorité nécessaire à cet effet passe des deux tiers aux trois-quarts. Enfin, les ébauches voulaient que chaque groupe militant soit animé conjointement par un " maître des débats " (" Diskussionsleiter ") et deux délégués de l'échelon immédiatement supérieur. Le texte entériné le 7 octobre confère cette tâche aux Premier et Second Porte-Parole (" Erster » et "Zweiter Sprecher ») et aux deux délégués. Cette évolution appelle deux remarques. D’une part, près du tiers des membres d'un maillon donné est doté d'un mandat, ce qui revient à responsabiliser les intéressés. D'autre part, le pouvoir est réparti sur quatre personnes, d'autant que la terminologie implique que les mandataires exercent un rôle plus représentatif qu'exécutif ${ }^{20}$. Autrement dit, elle privilégie le fonctionnement horizontal typique des mouvements citoyens à la hiérarchie des partis classiques que sont, chacun à leur manière, le SED et le SPD de part et d'autre du Mur. Sans laisser d'être fortement teintés de l'héritage des mouvements citoyens, les statuts adoptés le 7 octobre 1989 portent donc la trace d'une inflexion qui va dans le sens du renforcement des mandats, conformément à la volonté initiale de Meckel et Gutzeit21.

der DDR. Vorläufiges Statut. Entwurf 2 » [«Entwurf 2 »], les deux ébauches figurent dans les archives privées de K. Elmer-Herzig (KEH).

16. Fin septembre, ce groupe est constitué des pasteurs Markus Meckel, Martin Gutzeit, Arndt Noack, Konrad Elmer, Rainer Hartmann, Steffen Reiche, Jochen Goertz, Reiner Rühle, de la biologiste sans emploi Angelika Barbe et du maçon puis bibliothécaire et agent officieux du MfS, Manfred « Ibrahim » Böhme.

17. Cf. entretien avec Elmer-Herzig, Potsdam, le 23 juin 2011 et ELMER, « Vor- und Wirkungsgeschichte... », op. cit., p. 30 sq.

18. Cf. MECKEL, "Programmatischer Vortrag... », op. cit., p. 379-396.

19. Cf. WEIDEN, « Das Profil der Sozialdemokratischen Partei... », op. cit., p. 49.

20. Cf. « Entwurf 1 » et " Entwurf 2 », articles 6, 7, 8 et 10 et « Statut 1 », articles 11, 14, 15, 17 et 18.

21. Cf. les entretiens avec Elmer-Herzig et Gutzeit, à Berlin, le 21 mars 2011. 
Partant, on pourrait être tenté de conclure à une concentration du pouvoir entre les mains de quelques élus du parti. Or, le SDP entend délibérément démocratiser la société et non pas se substituer purement et simplement au SED dans ce qui serait une révolution de palais. Ainsi, pour contrebalancer le pouvoir des mandataires, il est fait une place de choix à la base qui doit servir de point de départ de la construction effective du parti. Le nombre des échelons subalternes au Directoire national ("Republikvorstand ») doit dépendre du nombre d'adhérents puisque chacun ne peut compter plus de quatorze membres ou délégués. De fait, on procède d'abord à la création du Directoire national puis des directoires régionaux avant de combler le vide institutionnel entre les groupes de base et les instances exécutives ${ }^{22}$. II convient néanmoins de retenir qu'intellectuellement, les pères des statuts sacrifient au principe de rehausser la base, ethos hérité des mouvements citoyens et de leur expérience de l'autoritarisme du régime. Par ailleurs, la structuration du parti renvoie à la tradition sociale-démocrate. Le SDP s'enracine dans des sections, organisées par répartition géographique, et non pas dans des cellules d'entreprises, d'inspiration communiste. Dans les termes, ces "groupes de base " (" Basisgruppe ») font néanmoins référence à la démocratie par la base qui anime les mouvements citoyens, et non pas à la nomenclature qui a cours dans le SPD ouest-allemand ("Ortsverein " ou "Abteilung "). On peut voir dans ce choix un refus de lui faire allégeance. Notons à cet égard que les statuts déclarent l'organisation " solidaire des partis socialistes et sociaux-démocrates européens " sans faire une place de choix au SPD qui n'est pas mentionné expressément ${ }^{23}$. Quoi qu'il en soit, la valorisation de la démocratie par la base laisserait présager une valorisation des congrès et ils sont bien déclarés organe suprême. L'évolution des texte-cadre dénote pourtant une réticence croissante à leur égard : les ébauches en prescrivaient la tenue annuelle à chaque échelon, tandis que les "Statut 1 " n'en prévoient qu'à partir de l'hypothétique niveau cantonal ("Kreisebene "). II leur revient de fixer les lignes directrices du parti qui s'appliquent, après consultation, aux niveaux inférieurs. À côté de ces congrès, les statuts prévoient que soit élu un conseil exécutif de quatorze personnes choisies prioritairement selon le principe de représentativité : chaque entité délègue l'un de siens ${ }^{24}$. Afin de limiter le pouvoir de ces Conseils, la convocation de congrès extraordinaires est facilitée puisqu'elle requiert le vote du tiers des groupes représentés ou des délégués au lieu du double. Enfin, ultime garde-fou contre une autonomisation des instances de décision vis-à-vis de la base, chaque élu est responsable devant sa base. Il est tenu de rendre compte de ses décisions et des débats qui animent les instances dans lesquelles il siège ${ }^{25}$.

Les premiers statuts sociaux-démocrates satisfont à une double exigence. D'une part, le système élaboré des contre-pouvoirs place le SDP aux antipodes du SED dont il rejette le « centralisme démocratique ", c'est-à-dire la concentration, dictatoriale selon lui, des pouvoirs. D'autre part, le choix du parti permet de munir des mandataires élus et responsables de leur action de mandats forts qui leur permettent d'exécuter des décisions

22. Cf. GUTZEIT, Martin / HEIMANN, Siegfried (éd.), Die Gründungsversammlung. Die Gründung des Berliner Bezirksverbandes der SDP am 5.11.1989 in der Sophienkirche in Berlin. Gekürztes Wortprotokoll nach einer Abschrift von einer achtstündigen Videoaufzeichnung, Veröffentlichungen der Historischen Kommission der SPD, Berlin, 1999, (KEH).

23. Cf. "Statut 1 », article 2. W. Brandt et H. Schmidt n'en sont pas moins des figures tutélaires des sociauxdémocrates est-allemands dès avant la chute du Mur, cf. par exemple les entretiens à Berlin avec HJ. Hacker le $1^{\text {er }}$ avril 2011, K. Herbst le 23 janvier 2012 et St. Reiche le 30 mars 2011.

24. La question des majorités n'entre en considération que s'il reste des mandats vacants, une fois les quotas remplis, pour atteindre le nombre total de 14 membres cf. "Statut 1 », article 16. Les Conseils n'étaient pas envisagés dans les ébauches.

25. Sur tous ces points, cf. « Entwurf 1 », « Entwurf 2 », articles 14, 16, 20 et « Statut 1 », articles 16, $21,23$. 
collectives. Néanmoins, ce parti ne renie pas en bloc l'héritage des mouvements citoyens desquels il reste solidaire dans l'action. Les sociaux-démocrates de la première heure tentent bien plutôt la synthèse entre le caractère éminemment vertical du SED et les structures largement horizontales des mouvements. Aussi l'identité du parti socialdémocrate en RDA est-elle, tout comme ses membres fondateurs, fortement marquée par l'opposition aux structures de domination propres à la RDA et par le contexte immédiat de l'automne 1989.

\section{Enjeux de pouvoir interne}

Lorsqu'en février 1990, le premier Congrès régulier ("Parteitag ») du désormais SPD-Est est appelé à entériner les seconds statuts ("Statut 2 ") 26 à Leipzig, la situation politique du pays et du parti est tout autre. Il compte une bonne trentaine de milliers d'adhérents qui, le 13 janvier, lors de la Conférence des délégués ("Delegiertenkonferenz »), a imposé au premier Directoire élu le 7 octobre la modification du sigle en gage de proximité avec le grand frère ouest-allemand ${ }^{2728}$. Les membres fondateurs avaient de leur côté souhaité afficher leur indépendance quant à celui-ci en adoptant un sigle et un nom divergents qui signifiaient en outre leur volonté d'inscrire leur action dans le cadre autonome de l'État estallemand. Plus significatif pour notre propos est le rapprochement effectif entre les deux entités socialesdémocrates, car Bonn envoie des permanents à Berlin dans le but officiel d'aider les néophytes est-allemands. Autant l'aide matérielle apportée par la centrale ouestallemande, la Erich-Ollenhauer-Haus, est la bienvenue, autant les membres fondateurs du SDP A. Barbe et J. Richter gardent le souvenir d'une prise en main plutôt que d'une entraide fraternelle en ce qui concerne la rédaction des textes essentiels ${ }^{29}$. Enfin, le parti a acquis une reconnaissance de fait par un régime en pleine déliquescence.

Ces trois aspects contextuels laissent présager une " normalisation " au sens où la dynamique révolutionnaire serait retombée et où il s'agirait dorénavant pour le parti de se doter de structures capables de soutenir un gouvernement issu de ses rangs. La thèse de l'oligarchisation proposée en 1911 par Robert Michels à propos du SPD de l'Empire wilhelminien pourrait connaître une nouvelle actualité si l'on constatait une tendance à la concentration des pouvoirs entre les mains des mandataires ${ }^{30}$. La collaboration croissante entre les deux homologues amène la question d'un éventuel rapprochement institutionnel qu'il convient d'étudier également.

Lorsque K. Elmer est appelé à la tribune pour présenter le texte soumis au congrès, il prend l'assistance à témoin pour contrer des tendances à l'oligarchisation qu'il décèle dans le comportement du Directoire auquel il appartient. II affirme en effet qu'auparavant, le Directoire proposait un élargissement de ses rangs à 40 mandataires et entendait coopter 50 autres personnalités pourvues du droit de vote. Aussi aurait-il comptabilisé jusqu'à 90 voix contre 150 délégués réguliers des fédérations subalternes. Dans la version défendue par Elmer, l'exécutif compte 33 membres, les personnes cooptées ne sont dotées que d'un

26. Cf. «Statut », in : VORSTAND DER SPD, Grundsatzprogramm. Statut, Berlin, 1990, p. 49-58, [" Statut 2 »].

27. VORSTAND DER SDP, Protokoll Delegiertenkonferenz der Sozialdemokratischen Partei in der DDR. 12.1.-

28 .1.1990. Berlin, Kongreßhalle, Alexanderplatz, Berlin, 1990, p. 114-139, in : 2/SDPA000007.

29. Cf. STURM, Uneinig in die Einheit..., op. cit., p. 166 et 273 et les entretiens avec A. Barbe à Dresde le 9 janvier 2012 et J. Richter à Berlin le 12 décembre 2011.

30. Cf. MICHELS, Robert, Zur Soziologie des Parteiwesens in der modernen Demokratie. Untersuchungen über die oligarchischen Tendenzen des Gruppenlebens, coll. Kröners Taschenbuchausgabe, 4e éd., Alfred Kröner Verlag, Stuttgart, 1989 [1911]. 
avis consultatif, et surtout, le nombre de délégués réguliers est porté à 300 . Les dispositions adoptées in fine se recoupent largement avec les statuts ouest-allemands, de sorte que l'occidentalisation des statuts sert ici à Elmer à contrecarrer la tendance avérée à l'oligarchisation ${ }^{31}$.

Le congrès de Leipzig vient considérablement modifier le texte-cadre précédent en ce qui concerne la structuration $d u$ bas vers le haut. Rappelons que le principe de représentativité prévalait puisque chaque élément subalterne devait déléguer deux de ses membres à l'échelon supérieur, la majorité relative ne jouant qu'en cas de postes restant à pourvoir pour atteindre le nombre de quatorze. Avec ce nouveau texte, c'est aux congrès des fédérations du même échelon de choisir les membres de l'exécutif - indépendamment de l'implantation des candidats. Le nombre de délégués n'est plus paritaire mais dépend du nombre d'adhérents que compte chaque fédération, ce qui revient à mettre les structures du parti en concurrence pour recruter un nombre maximal de membres. Enfin, la double structure des congrès et conseils, chère à Elmer, est abandonnée au profit des seuls premiers. Ici encore, l'évolution tend plus vers l'occidentalisation que vers l'oligarchisation ${ }^{32}$.

Les premiers statuts, on s'en souvient, prohibaient toute forme de collusion entre les instances de pouvoir du parti et de l'État. Alors que les sociaux-démocrates est-allemands sont donnés vainqueurs au scrutin législatif national du 18 mars, certains représentants de la première élite du SDP/SPD-Est, issue de Schwante, ont bon espoir de compter parmi les futurs députés de la Volkskammer voire d'obtenir un portefeuille ministériel. Meckel devient en effet ministre des Affaires étrangères du gouvernement de Maizière et député aux côtés de S. Leger, K. Elmer ou A. Barbe, pourtant tous signataires des textes originels. Ce cumul est rendu possible par l'adoption dans les "Statut 2 " d'une formule autrement moins contraignante : il ne doit pas être " excessif ${ }^{33}$. On pourrait être porté à croire qu'il s'agit d'un cas flagrant d'oligarchisation. Selon Weiden, il n'en est rien puisque l'interdiction est levée à l'initiative de la base qui ne rechigne guère à l'idée de s'inspirer du fonctionnement du parti ouestallemand, gage de succès électoraux ${ }^{34}$. Les membres fondateurs reviennent toutefois sur leurs positionnements d'origine au moment où les portes du pouvoir s'ouvrent à eux. Rien ne les empêche en effet de démissionner de leurs fonctions partisanes s'ils souhaitent accéder aux responsabilités étatiques.

Au sein même du parti, le cumul des mandats est dorénavant autorisé à condition qu'il n'y ait pas conflit d'intérêts : ainsi, un membre du Directoire ne saurait en même temps présider le congrès du même échelon ni siéger dans les Commissions de contrôle et d'arbitrage (Kontrollkommission, Schiedskommission), censées contrôler l'action de l'exécutif. Le Conseil du parti (Parteirat) demeure indépendant du Directoire qui peut assister à ses cessions mais est condamné au mutisme sauf sollicitation expresse de la part de son Président. On est loin des projets du Directoire qui s'octroyait, selon Elmer, le droit

31. Cf. ELMER, Konrad, "Statutendiskussion Leipziger Parteitag 22.2.90 ", Leipzig, 22 février 1990, p. 56. [" Statutendiskussion "], p. 57, "Statut 2 ", articles 12 et 18 et "Organisationsstatut " article 15. Les " Organisationsstatut " sont les statuts du SPD-ouest, en vigueur depuis 1971, et reproduits intégralement dans KNIGGE, Arnold, " Sitzung der Statutenkommission der DDR-SPD am 12. Juli 1990 in Berlin (Ost) », Bonn, 17 juillet 1990, in : [AdsD] 2/PVDZ000236.

32. Cf. "Statut 2 », article 12, "Organisationsstatut ", article 15.

33. "Es ist darauf zu achten, daß es nicht zu einer Ämterhäufung kommt. Insbesondere kann kein Vorstandsvorsitzender / keine Vorstandsvorsitzende den Vorsitz in zwei Vorständen haben. ». La spécification de la deuxième phrase annule la dimension contraignante de la première, cf. "Statut 2 ", article 27.

34. Cf. WEIDEN, « Das Profil der Sozialdemokratischen Partei... », op. cit., p. 143. 
d'y siéger de plein droit, mais proche des textes ouest-allemands. De ce fait, les hiérarchies se trouvent renforcées par rapport aux "Statuts 1 " mais beaucoup moins que ne le souhaiterait le premier Directoire, de sorte que la tendance à l'oligarchisation qui l'anime est contrecarrée par l'occidentalisation ${ }^{35}$.

Ces deux tendances se rejoignent en revanche dans la requalification des postes exécutifs. Le SPD-Est s'affranchit du milieu des mouvements citoyens et reprend la nomenclature plus verticale d'Allemagne de l'Ouest : le porte-parolat est remplacé par l'idée de présidence. Le Directoire national et son président ("Vorsitzender ») ont pour tâche de diriger le parti et de " prend[re] les dispositions transitoires nécessaires ». Parallèlement, il n'est plus fait mention de la responsabilité des élus face à leur base. De ce fait, l'exécutif se renforce considérablement tout en s'appuyant sur les textes ouest-allemands. Notons que les deux derniers points sont absents de l' « Organisationsstatut » et ne sont donc imputables qu'à l'oligarchisation est-allemande. Sur ce point, les compétences du Directoire sont plus étendues qu'à l'Ouest ${ }^{36}$.

Globalement, on le voit, le Parti social-démocrate est-allemand passe, en cinq mois, d'un groupuscule d'anciens activistes des mouvements citoyens à un parti de taille considérable, et se dote d'institutions beaucoup plus verticales. Ce changement de paradigme statutaire se fait souvent à l'initiative non pas des sociaux-démocrates de la première heure mais quoi qu'en dise Elmer - de cette base beaucoup plus hétérogène, venue grossir les rangs du parti. Elle est moins marquée par le fonctionnement des mouvements citoyens et moins attachée à la RDA en tant qu'État allemand de plein droit. N'oublions pas non plus que, depuis l'automne, le Mur est tombé et que le régime du Nationale Front est en plein délitement, autant d'événements qui commandent un repositionnement. Partant, cette base ne rechigne pas à suivre le modèle ouest-allemand et sacrifie certaines des spécificités du parti est-allemand pour s'aligner globalement sur le fonctionnement du SPD ouestallemand. À la démocratie par la base succède une autre forme de démocratie, celle représentative. II paraît néanmoins abusif de conclure comme Gröf à l'aboutissement du dessein initial - mais non avoué afin de ne pas rebuter d'éventuels adhérents de leur propre milieu - du groupe de préparation de la fondation autour de Meckel et de Gutzeit ${ }^{37}$.

\section{Maintenir une social-démocratie est-allemande en Allemagne de l'Est}

La question des statuts redevient brûlante quelques mois plus tard. Entre-temps, nous l'avons vu, le scrutin du 18 mars appelle la négociation rapide de l'unification allemande. La question de la fusion des partis s'en trouve posée par ricochet. Dès mai et juin 1990, on assiste à la mise en place de tandems entre Berlin et Bonn, à l'envoi croisé d'observateurs des sessions des Directoires nationaux et à l'instauration de trois commissions paritaires dont celle dédiée à la question statutaire. Parallèlement, Bonn entreprend le recensement des militants sociaux-démocrates en RDA et fixe comme horizon de fusion la tenue des prochaines élections législatives nationales en RFA tandis qu'à Berlin, on l'envisage d'ici dix-

35. Cf. "Statut 1 ", article 22, "Statut 2 ", articles 16, 22, 25 et 26, "Organisationsstatut ", articles 28 à 30 et « Statutendiskussion », p. 58.

36. Cf. " Statut 1 », articles 14 et 28, "Statut 2 », articles 10, 18 et 35 et " Organisationsstatut », article 23.

37. Cf. GRÖF, Wolfgang : "In der frischen Tradition des Herbstes 1989 ». Die SDP / SPD in der DDR : Von der Gründung über die Volkskammerarbeit zur deutschen Einheit, Beiträge aus dem Archiv der sozialen Demokratie, t. 1, AdsD der FES, $2^{\mathrm{e}}$ ed. augmentée, Bonn, 1996, p. 16. 
huit mois ${ }^{38}$. Après l'élection de Wolfgang Thierse à la tête du SPD-Est le 8 juin au congrès de Halle, les efforts s'accélèrent, tant il est vrai que celui-ci, " révolutionnaire de novembre " selon le bon mot de Sturm, ne fait pas partie des membres fondateurs et n'affiche pas le même attachement à l'héritage est-allemand que ces derniers. Pour la base du parti estallemand, cette spécificité est une qualité, de sorte que son élection est emblématique du divorce croissant entre les sociaux-démocrates de la première heure et la base ${ }^{39}$.

Le 17 juin, en marge des festivités autour de la fête nationale ouest-allemande, les membres du Directoire est-allemand St. Hilsberg, K.A. Kamilli, H. Kuessner, H. Ringstorff et W. Thierse se réunissent avec leurs homologues H. Däubler-Gmelin, A. Fuchs, H.U. Klose, D. Stobbe, H.J. Vogel et les permanents A. Berndt, A. Knigge et P. Weber. Le Président ouestallemand propose l'adoption de quotas provisoires pour donner plus de poids aux délégués est-allemands. L'article 15 de l'« Organisationsstatut » qui dispose que leur nombre est fonction de celui des militants qu'ils représentent, s'en trouverait momentanément suspendu ${ }^{40}$. En contrepartie, Vogel cherche à obtenir un élargissement des structures ouestallemandes au parti est-allemand plutôt que leur refonte. II argue de ce que le calcul du nombre de délégués est-allemands serait d'autant plus difficile s'il fallait renouveler le Directoire dans son économie. Le Secrétaire général du SPD-Est, Hilsberg, ne se montre guère impressionné par cette menace à peine voilée et revendique une minorité de blocage de $35 \%$ pour les siens. La question n'est pas tranchée ${ }^{41}$. En revanche, cette entrevue fait des statuts du

SPD ouest-allemand la base des négociations entre les deux partis. On peut y voir un parallèle avec la réalisation de l'unification nationale à partir de l'article 23 et non pas de l'article 146 de la Loi fondamentale qui aurait débouché, lui, sur une nouvelle Allemagne ${ }^{42}$. Ajoutons qu'un vote de la base du SPD, théoriquement nécessaire en cas de fusion ou de dissolution du SPD-Ouest est écarté le 25 juin au moyen d'une astuce juridique : plutôt que de parler de "fusion ", il s'agit de rétablir l'" état normal de la social-démocratie en Allemagne » par un simple vote en congrès d'une proclamation allant dans ce sens ${ }^{43}$. Le SPDOuest opère un véritablement changement de paradigme faisant de la période allant de 1946 à 1990 une parenthèse aberrante qu'il convient de refermer au plus vite. Par ricochet, il balaie l'identité du SPD-Est et les mérites révolutionnaires de ses militants, notamment ceux de la première heure.

Selon les projets ouest-allemands, l'élargissement du SPD-Ouest à son homologue estallemand doit se faire en trois temps selon les dispositions du nouvel article 41 : l'alinéa a)

38. Cf. HILSBERG, Stephan, "Gespräche Bonn ", [Berlin], 31 mars 1990, in : [AdsD] 2/SDPA000187 et LODE, Jutta, " Protokoll der Präsidiumssitzung am 11. 4. 1990 », Berlin, 11 avril 1990, in : [AdsD] 2/SDPA000070, HILSBERG, Stephan, "Vereinbarungen nach dem Gespräch zwischen Anke Fuchs und Stephan Hilsberg am 2. Mai 1990 in Ost-Berlin ", [Berlin], [mai 1990], BORKENHAGEN, Franz / KNIGGE, Arnold / WARDIN, Peter, "Vermerk für Anke Fuchs, MdB », Bonn, 30 mai 1990, tous deux in : [AdsD] 2/SDPA000187 et [Anonyme], " Personelle Besetzung d. Statutenkommission It. Vorstandssitzung v. 18.06.90 ", [Berlin], 18 juin 1990, in : [AdsD] 2/SDPA000164.

39. Cf. STURM, Uneinig in die Einheit..., op. cit., p. 329.

40. Cf. "Organisationsstatut », article 15.

41. Cf. KNIGGE, Arnold, "Sitzung der Gemeinsamen Kommission zur Parteivereinigung am 17. juni 1990 », Bonn, 28 juin 1990, in : [AdsD] 2/SDPA000188.

42. S ur les positionnements du SPD-Est quant aux Articles 23 et 146 de la Loi fondamentale, cf. VORSTAND DER SPD, « Ja zur deutschen Einheit - eine Chance für Europa. Wahlprogramm der SPD zum ersten frei gewählten Parlament der DDR », Berlin, [février] 1990 et STURM, Uneinig in die Einheit..., op. cit., p. 430-435.

43 . C f. [VORSTAND DER SPD-(WEST)], « Änderung des Organisationsstatuts und der Finanzordnung zur Wiederherstellung der Einheit der Sozialdemokratischen Partei Deutschlands ", [Bonn], 25 juin 1990, in 2/SDPA000185 et SPD, " Manifest zur Wiederherstellung der Einheit der Sozialdemokratischen Partei Deutschlands », 27 septembre 1990. 
assure l'intérim jusqu'au jour de l'Unification allemande, les alinéas b) à e) régissent la période jusqu'à la tenue du prochain congrès régulier, prévu en 1991, et enfin l'alinéa f) couvre celle allant jusqu'au suivant, en 1993. La première disposition invite 100 délégués est-allemands à s'adjoindre aux 400 délégués de l'Ouest pour entériner la fusion et met les deux Directoires nationaux sur un pied d'égalité pour la semaine entre le 27 septembre et le 3 octobre qui consacre l'unité allemande ${ }^{44}$. Avec $20 \%$ des votants, les Allemands de l'Est sont loin d'atteindre la minorité de blocage revendiquée par Hilsberg mais demeurent en surnombre puisqu'ils représentent chacun 415 membres contre 2250 à l'Ouest. Une application stricte de l'article 15 des " Organisationsstatut » aurait divisé leur nombre par cinq.

Pour la période intermédiaire, l'article 41 crée un poste supplémentaire de VicePrésident, dix nouveaux postes au Directoire et autant au Bureau qui sont à pourvoir parmi les seuls sociaux-démocrates est-allemands le 26 septembre. La menace de Vogel semble avoir porté ses fruits puisqu'ainsi, les Allemands de l'Ouest n'ont pas à remettre en jeu leurs mandats exécutifs avant échéance. En 1991, ces postes supplémentaires sont maintenus mais tout social-démocrate allemand est dorénavant éligible. Parallèlement, le nombre de délégués provenant des fédérations régionales est-allemandes est fonction du nombre de membres et de scrutins qu'elles totalisent. Enfin, en 1993, le nombre total de délégués de 1991 est à reconduire selon une stricte représentativité ${ }^{45}$. Les Allemands de l'Est tentent en vain de pérenniser les quotas de 1991 jusqu'en 1995. Autrement dit, la Erich-OllenhauerHaus concède aux Allemands de l'Est une surreprésentation éphémère qui ne fait que retarder la dissolution de l'influence est-allemande dans les structures unifiées étant donné la prépondérance numérique des sociaux-démocrates ouest-allemands. Plus généralement, Bonn parvient à imposer son fonctionnement aux structures est-allemandes. On serait tenté de conclure que le SPD-Ouest a simplement happé le parti frère de RDA.

Pourtant, des éléments proprement est-allemands figurent jusqu'aujourd'hui dans I'“ Organisationsstatut » de 2014. Elmer se montre fier d'avoir imposé, avec l'aide de Vogel, précise-t-il, la reprise quasi intégrale du préambule est-allemand. La notion de la démocratie par la base est, quant à elle, introduite dans l'article $8^{46}$. À l'en croire, Vogel se révèle un allié de choix pour les sociaux-démocrates est-allemands tant qu'ils ne cherchent pas à remettre en cause les assises de son pouvoir ${ }^{47}$. En revanche, les tentatives d'Elmer d'ancrer dans les futurs statuts communs l'idée de scinder les sections pour garantir la possibilité de débats, d'interdire le cumul de mandats ou encore, mesure toute symbolique, de faire de Berlin le seul siège du SPD unifié restent infructueuses ${ }^{48}$.

Avec le recul, Elmer n'en juge pas moins en 1992 que la marque des membres fondateurs du SDP est plus tangible en matière statutaire que programmatique ${ }^{49}$. Quelques années plus tard, alors qu'il n'est plus député du Bundestag mais que le chancelier est social-démocrate, le pasteur Elmer en appelle à l'actualisation du principe de la démocratie par la base contre

44. Cf. [VORSTAND DER SPD-(WEST)], « Änderung des Organisationsstatuts... ».

45. C f. « Organisationsstatut », articles 15, 23 et 28, [VORSTAND DER SPD-(WEST)], « Änderung des Organisationsstatuts... ".

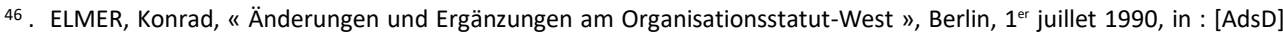
2/SDPA000188, p. 36 sq. et « Statut 2 », Präambel et Article 8 et ELMER, « Vor- und Wirkungsgeschichte... », p. 36 sq

47. Par ailleurs, les membres fondateurs du SDP tombent d'accord pour voir en Vogel leur meilleur allié ouestallemand avec Brandt cf. les entretiens avec K. Elmer-Herzig, M. Meckel, St. Reiche.

48. E LMER, « Änderungen und Ergänzungen am Organisationsstatut-West », ELMER, " Vor- und Wirkungsgeschichte... », p. 36 sq., " Statut 2 », Article 9 et « Organisationsstatut », articles 1 et 8.

49. ELMER, « Vor- und Wirkungsgeschichte... », p. 38. 
l'élite de son propre parti. Cet appel concerne les aspects politiques et non plus structurels : « [...] Article 8, alinéa 1 : La "volonté politique se cristallise du bas vers le haut." Ce ne serait pas un mal si, vingt ans après la création, la base nombreuse rappelait cette phrase au bon souvenir de l'élite peu nombreuse ${ }^{50}$.

L'arrivée des sociaux-démocrates est-allemands n'a pas révolutionné le parti socialdémocrate d'Allemagne, ni en matière programmatique, ni en matière statutaire. En revanche, l'émergence du SDP puis l'institutionnalisation du parti est-allemand ont contribué à profondément modifier la RDA en proposant une troisième voie politique dans le cadre révolutionnaire de l'automne 1989 puis une troisième voie entre les socialistes convaincus de la supériorité morale de la RDA et de son régime et ceux s'enthousiasmant pour une unification à toute vapeur, au prix d'une reddition complète de l'identité propre des Allemands de l'Est qui s'est construite au cours de quarante ans. Ces quelques pages ont tenté de montrer que l'évolution statutaire du SDP/ SPD-Est est le reflet de cette lutte.

50. E LMER-HERZIG, Konrad, " Auf den Anfang kommt es an ! ", http://www.tagesspiegel.de/themen/mein-jahr89themenseite/ost-spd-auf-den-anfang-kommt-es-an/1624380.html [20 février 2017]. 